\title{
A TRANSITIVE SELF-POLAR DOUBLE-TWENTY OF PLANES
}

\section{P. B. KIRKPATRICK}

(Received 25 May 1994; revised 8 January 1995)

Communicated by R. Howlett

\begin{abstract}
We demonstrate the existence, in the 5-dimensional projective space over any field $\mathscr{F}$ in which $1+1 \neq 0$ and -1 is a square, of a non-degenerate double-twenty of planes $(\mathscr{H}, \mathscr{K})$ with the property that there is a group of collineations which acts transitively on $\mathscr{H} \cup \mathscr{K}$ while each element of the group either maps $\mathscr{H}$ onto itself and $\mathscr{K}$ onto itself or else swaps $\mathscr{H}$ with $\mathscr{K}$. If there is an involutory automorphism of $\mathscr{F}$ which swaps the two square roots of -1 , then $(\mathscr{H}, \mathscr{K})$ is also self-polar (with respect to a unitary polarity). We describe some of the geometry (in both 5-dimensional and 3-dimensional space) associated with the double-twenty $(\mathscr{H}, \mathscr{K})$ and its group.
\end{abstract}

1991 Mathematics subject classification (Amer. Math. Soc.): primary 51A45.

\section{Introduction}

1.1. By a double-twenty of planes we mean a pair $\left(\mathscr{H}^{\circ}, \mathscr{K}^{\circ}\right)$, where each of $\mathscr{H}^{\circ}$ and $\mathscr{K}^{\circ}$ is a set of planes in the same 5-dimensional projective space, $\left|\mathscr{H}^{\circ}\right|=\left|\mathscr{K}^{\circ}\right|=20$ and there is a bijection $\pi_{\circ}: \mathscr{H}^{\circ} \longrightarrow \mathscr{K}^{\circ}$ (called the pairing map) such that, for all $H$ in $\mathscr{H}^{\circ}$ and $K$ in $\mathscr{K}^{\circ}, H$ intersects $K$ if and only if $H \pi_{\circ} \neq K$. The double-twenty of planes, like the better known double-six of lines in 3-dimensional projective space, arises naturally in the theory of determinantal varieties. For a detailed account of this connection, see Room [5, pp. 72-81 and pp. 429-433].

1.2. If $\left(\mathscr{H}^{\circ}, \mathscr{K}^{\circ}\right)$ is a double-twenty of planes and $\mathscr{H}^{\circ} \cap \mathscr{K}^{\circ} \neq \emptyset$, then we call $\left(\mathscr{H}^{\circ}, \mathscr{K}^{\circ}\right)$ a degenerate double-twenty. A degenerate double-twenty of planes can be constructed in any 5 -dimensional projective space as follows. Choose six points which do not lie in the same hyperplane. These points determine twenty planes, each plane passing through three of the six points. Label these twenty planes $H_{1}, \ldots, H_{20}$.

(C) 1996 Australian Mathematical Society $0263-6115 / 96 \$ A 2.00+0.00$ 
Also label them $K_{1}, \ldots, K_{20}$ in such a way that (for $i=1, \ldots, 20$ ) $K_{i}$ does not intersect $H_{i}$. Then $\left(\left\{H_{1}, \ldots, H_{20}\right\},\left\{K_{1}, \ldots, K_{20}\right\}\right)$ is a degenerate double-twenty.

1.3. We shall say that a double-twenty of planes $\left(\mathscr{H}^{\circ}, \mathscr{K}^{\circ}\right)$ is transitive if the group, consisting of all those collineations (of the enveloping 5-dimensional projective space) which either map $\mathscr{H}^{\circ}$ onto itself and $\mathscr{K}^{\circ}$ onto itself or else swap $\mathscr{H}^{\circ}$ and $\mathscr{K}^{\circ}$, acts transitively on the set $\mathscr{H}^{\circ} \cup \mathscr{K}^{\circ}$. The degenerate double-twenty of planes described in the previous paragraph is an example of a transitive double-twenty. However, we are naturally more interested in the non-degenerate case.

1.4. If $\left(\mathscr{H}^{\circ}, \mathscr{K}^{\circ}\right)$ is a double-twenty of planes with pairing map $\pi_{\circ}$ then we say that $\left(\mathscr{H}^{\circ}, \mathscr{K}^{\circ}\right)$ is self-polar if there is a polarity $\alpha_{\circ}$ (of the enveloping 5-dimensional projective space) such that $H \alpha_{\circ}=H \pi_{\circ}$ for all $H$ in $\mathscr{H}^{\circ}$. Note that, whereas the polarities discussed in $[3,4$ and 6$]$ are all orthogonal, the polarity referred to in Section 5 of this paper is unitary.

1.5. In Section 2 we demonstrate the existence of a transitive non-degenerate doubletwenty of planes $(\mathscr{H}, \mathscr{K})$ in the 5 -dimensional projective space $P G(5, \mathscr{F})$ over any field $\mathscr{F}$ in which $1+1 \neq 0$ and -1 is a square.

We do this by exhibiting a matrix $\mathbf{M}$ which defines a certain determinantal quartic hypersurface $\mathscr{D}$ in $P G(5, \mathscr{F})$ on which the required double-twenty of planes $(\mathscr{H}, \mathscr{K})$ is situated. Examining the equation $\operatorname{det}(\mathbf{M})=0$ of the hypersurface $\mathscr{D}$, we identify certain projective collineations $a, b, c, r$ and $j$ of $P G(5, \mathscr{F})$ which map $\mathscr{D}$ onto itself. We then show how the action of these collineations on the set $\mathscr{H} \cup \mathscr{K}$ may be described in terms of the action of certain projective collineations of $P G(3, \mathscr{F})$ on a certain set $\mathbf{S}$ of points in $P G(3, \mathscr{F})$. The simplicity and symmetry of the equation of $\mathscr{D}$ enable us to actually calculate (without undue difficulty) the vectors representing the points in $\mathbf{S}$, giving us explicit equations for each of the forty planes in $\mathscr{H} \cup \mathscr{K}$. Using these equations and facts about the action of the group $\mathscr{G}=\langle a, b, c, r, j\rangle$ on $\mathscr{H} \cup \mathscr{K}$ obtained by studying the action on $\mathbf{S}$ of suitable collineations in $P G(3, \mathscr{F})$, we conclude that $(\mathscr{H}, \mathscr{K})$ is a transitive non-degenerate double-twenty.

1.6. In Sections 3 and 4 , we describe the geometry of the sets $\mathbf{S}$ and $\mathscr{H} \cup \mathscr{K}$, and also the actions of the relevant collineation groups on these sets, in some detail; while in Section 5 we observe that, if the coordinate field $\mathscr{F}$ possesses an involutory automorphism which swaps the two square roots of -1 , then the double-twenty $(\mathscr{H}, \mathscr{K})$ is not only transitive but also self-polar (with respect to a unitary polarity which commutes with every element of $\mathscr{G}$ ).

1.7. Definitions for some of the terms used, but not defined, in this paper can be found in [1, Section 1.4] and [7, Chapter 10]. Note, however, that what we call a projective space is in [1] called a projective geometry. 


\section{A transitive non-degenerate double-twenty}

2.1. The matrix M. Suppose that $\mathscr{F}$ is any field in which $1+1 \neq 0$ and -1 is a square, and that $i$ is one of the two square roots of -1 in $\mathscr{F}$. Now consider the (commutative) polynomial ring $\mathscr{R}=\mathscr{F}[x, u, y, v, z, w]$, where $x, u, y, v, z, w$ are algebraically independent over $\mathscr{F}$. The indeterminates $x, u, y, v, z, w$ may be regarded as homogeneous coordinate variables for the 5-dimensional projective space $\operatorname{PG}(5, \mathscr{F})$ over $\mathscr{F}$. We are interested in a certain matrix over $\mathscr{R}$, namely the matrix

$$
\mathbf{M}=\left(\begin{array}{cccc}
0 & z & -v & -x \\
-w & 0 & x & -y \\
y & -u & 0 & -z \\
u & v & w & 0
\end{array}\right)
$$

Let

$$
\begin{aligned}
& D(x, u, y, v, z, w)=\operatorname{det}(\mathbf{M}) \\
& \quad=x^{2} u^{2}+y^{2} v^{2}+z^{2} w^{2}+\left(x^{2}+u^{2}\right) y v+\left(y^{2}+v^{2}\right) z w+\left(z^{2}+w^{2}\right) x u .
\end{aligned}
$$

Then $D(x, u, y, v, z, w)=0$ is the equation of a determinantal quartic hypersurface $\mathscr{D}$ in $P G(5, \mathscr{F})$, and it is easily seen that

$$
\begin{aligned}
D(x, u, y, v, z, w) & =D(u, x, y, v,-w,-z)=D(u, x, i y,-i v,-w, z) \\
& =D(y, v, z, w, x, u)=D(u, x, v, y, w, z) .
\end{aligned}
$$

It is less obvious, but can be checked, that $D(x, u, y, v, z, w)=D\left(x^{\prime}, u^{\prime}, y^{\prime}, v^{\prime}, z^{\prime}, w^{\prime}\right)$, where $\left(x^{\prime}, u^{\prime}, y^{\prime}, v^{\prime}, z^{\prime}, w^{\prime}\right)=(x, u, y, v, z, w) \mathbf{a}$, and

$$
\mathbf{a}=\frac{1}{2}\left(\begin{array}{cccccc}
0 & 0 & 1 & 1 & -1 & 1 \\
0 & 0 & 1 & 1 & 1 & -1 \\
1 & 1 & -1 & 1 & 0 & 0 \\
1 & 1 & 1 & -1 & 0 & 0 \\
-1 & 1 & 0 & 0 & 1 & 1 \\
1 & -1 & 0 & 0 & 1 & 1
\end{array}\right)
$$

2.2. The collineations $a, b, c, r$ and $j$. If $\mathbf{p}$ is a non-singular $6 \times 6$ matrix over $\mathscr{F}$ then by the projective collineation with matrix $\mathbf{p}$ we shall mean the collineation $p$ of $P G(5, \mathscr{F})$ which, for all non-zero $\mathbf{X}_{\circ}=\left(x_{\circ}, u_{\circ}, y_{\circ}, v_{\circ}, z_{\circ}, w_{\circ}\right)$ in $\mathscr{F}^{6}$, maps the point represented by the vector $\mathbf{X}_{\circ}$ to the point represented by the vector $\mathbf{X}_{\circ} \mathbf{p}$. Note that $p$ maps the hyperplane with equation $\mathbf{X} \mathbf{X}_{\circ}{ }^{t}=0$, where $\mathbf{X}=(x, u, y, v, z, w)$, to the hyperplane with equation $\left(\mathbf{X p}^{-1}\right) \mathbf{X}_{\circ}{ }^{t}=0$. 
From the equations in Section 2.1 we deduce that the hypersurface $\mathscr{D}$ is mapped onto itself by (each of) the projective collineations $a, b, c, r$ and $j$ with matrices $\mathbf{a}, \mathbf{b}, \mathbf{c}, \mathbf{r}$ and $\mathbf{j}$ (respectively), where $\mathbf{a}$ is as in Section 2.1 and

$$
\begin{array}{rlrl}
\mathbf{b} & =\left(\begin{array}{cccccc}
0 & 1 & 0 & 0 & 0 & 0 \\
1 & 0 & 0 & 0 & 0 & 0 \\
0 & 0 & 1 & 0 & 0 & 0 \\
0 & 0 & 0 & 1 & 0 & 0 \\
0 & 0 & 0 & 0 & 0 & -1 \\
0 & 0 & 0 & 0 & -1 & 0
\end{array}\right), & \mathbf{c}=\left(\begin{array}{cccccc}
0 & 1 & 0 & 0 & 0 & 0 \\
1 & 0 & 0 & 0 & 0 & 0 \\
0 & 0 & i & 0 & 0 & 0 \\
0 & 0 & 0 & -i & 0 & 0 \\
0 & 0 & 0 & 0 & 0 & 1 \\
0 & 0 & 0 & 0 & -1 & 0
\end{array}\right), \\
\mathbf{r}=\left(\begin{array}{cccccc}
0 & 0 & 0 & 0 & 1 & 0 \\
0 & 0 & 0 & 0 & 0 & 1 \\
1 & 0 & 0 & 0 & 0 & 0 \\
0 & 1 & 0 & 0 & 0 & 0 \\
0 & 0 & 1 & 0 & 0 & 0 \\
0 & 0 & 0 & 1 & 0 & 0
\end{array}\right), & \mathbf{j}=\left(\begin{array}{cccccc}
0 & 1 & 0 & 0 & 0 & 0 \\
1 & 0 & 0 & 0 & 0 & 0 \\
0 & 0 & 0 & 1 & 0 & 0 \\
0 & 0 & 1 & 0 & 0 & 0 \\
0 & 0 & 0 & 0 & 0 & 1 \\
0 & 0 & 0 & 0 & 1 & 0
\end{array}\right),
\end{array}
$$

2.3. The sets $\mathscr{H}$ and $\mathscr{K}$. If $U$ is a non-zero element of $\mathscr{F}^{4}$ then $U \mathbf{M}$ is a linear combination (over $\mathscr{F}$ ) of the rows of $\mathbf{M}$, and the subspace $U \mathbf{M}=O$ of $P G(5, \mathscr{F})$ lies on $\mathscr{D}$. Similarly, if $V$ is a non-zero element of $\mathscr{F}^{4}$ then $\mathbf{M} V^{t}$ is a linear combination of the columns of $\mathbf{M}$, and the subspace $\mathbf{M} V^{t}=O$ lies on $\mathscr{D}$. Since $U \mathbf{M}$ and $\mathbf{M} V^{t}$ both have four entries, the subspaces $U \mathbf{M}=O$ and $\mathbf{M} V^{t}=O$ both have (projective) dimension greater than or equal to one.

Let us denote by $\mathscr{H}$ the set of all planes on $\mathscr{D}$ whose equations may be written in the form $U \mathbf{M}=O$, and by $\mathscr{K}$ the set of all planes on $\mathscr{D}$ whose equations may be written in the form $\mathbf{M} V^{t}=O$ (where $U$ and $V$ belong to $\mathscr{F}^{4}$ ). (We are led to consider these two sets of planes by the general theory expounded in [5, pp. 72-73].)

2.4. The matrices $\mathbf{A}, \mathbf{B}, \mathbf{C}$ and R. Suppose that $H$ belongs to $\mathscr{H}$, that $K$ belongs to $\mathscr{K}$ and that $p$ is a projective collineation of $P G(5, \mathscr{F})$, with matrix $\mathbf{p}$, which maps $\mathscr{D}$ onto itself. Then $H p$ and $K p$ are planes on $\mathscr{D}$ and their equations may be obtained from the equations $U \mathbf{M}=O$ and $\mathbf{M} V^{t}=O$ of $H$ and $K$ by making the substitution $\mathbf{X} \longmapsto \mathbf{X p}^{-1}$, where $\mathbf{X}=(x, u, y, v, z, w)$.

It turns out that when the substitutions $\mathbf{X} \longmapsto \mathbf{X a}^{-1}, \mathbf{X} \longmapsto \mathbf{X b}^{-1}, \mathbf{X} \longmapsto \mathbf{X c}^{-1}$, $\mathbf{X} \longmapsto \mathbf{X r}^{-1}$ and $\mathbf{X} \longmapsto \mathbf{X j}^{-1}$ are applied to $\mathbf{M}$, the resulting matrices are (respectively) $-\mathbf{A M A}^{t},-\mathbf{B M B}^{t},-\mathbf{C}^{-1} \mathbf{M} \mathbf{C}^{t}, \mathbf{R} \mathbf{M R}^{t}$ and $-\mathbf{M}^{t}$, where

$$
\mathbf{A}=\frac{1}{2}\left(\begin{array}{cccc}
-1 & -1 & 1 & 1 \\
-1 & 1 & -1 & 1 \\
1 & -1 & -1 & 1 \\
1 & 1 & 1 & 1
\end{array}\right), \quad \mathbf{B}=\left(\begin{array}{llll}
0 & 0 & 0 & 1 \\
0 & 0 & 1 & 0 \\
0 & 1 & 0 & 0 \\
1 & 0 & 0 & 0
\end{array}\right)
$$




$$
\mathbf{C}=\left(\begin{array}{cccc}
0 & 0 & 0 & 1 \\
0 & 0 & i & 0 \\
0 & 1 & 0 & 0 \\
-i & 0 & 0 & 0
\end{array}\right), \quad \mathbf{R}=\left(\begin{array}{cccc}
0 & 0 & 1 & 0 \\
1 & 0 & 0 & 0 \\
0 & 1 & 0 & 0 \\
0 & 0 & 0 & 1
\end{array}\right)
$$

We deduce that the planes $H a, H b, H c, H r$ and $H j$ are given by the equations $(U \mathbf{A}) \mathbf{M}=O,(U \mathbf{B}) \mathbf{M}=O,\left(U \mathbf{C}^{-1}\right) \mathbf{M}=O,(U \mathbf{R}) \mathbf{M}=O$ and $\mathbf{M} U^{t}=O$ (respectively); and that the planes $K a, K b, K c, K r$ and $K j$ are given by the equations $\mathbf{M}(V \mathbf{A})^{t}=O, \mathbf{M}(V \mathbf{B})^{t}=O, \mathbf{M}(V \mathbf{C})^{t}=O, \mathbf{M}(V \mathbf{R})^{t}=O$ and $V \mathbf{M}=O$ (respectively).

It follows that $H a, H b, H c$ and $H r$ belong to $\mathscr{H}$, that $K a, K b, K c$ and $K r$ belong to $\mathscr{K}$, that $H j$ belongs to $\mathscr{K}$ and that $K j$ belongs to $\mathscr{H}$.

2.5. The set $\mathbf{S}$. Let us denote by $\mathbf{S}$ the set of all points $P$, in the 3-dimensional projective space $P G(3, \mathscr{F})$ over $\mathscr{F}$, such that if $U$ is a vector in $\mathscr{F}^{4}$ representing $P$ then the subspace $U \mathbf{M}=O$ of $P G(5, \mathscr{F})$ is a plane. (This method of representing the planes of $\mathscr{H}$ by points is a special case of the representation described in [5, pp. 79-81]).

Since $j$ swaps the subspaces $U \mathbf{M}=O$ and $\mathbf{M} U^{t}=O, \mathbf{S}$ may also be described as the set of all points $Q$ in $P G(3, \mathscr{F})$ such that if $V$ is a vector in $\mathscr{F}^{4}$ representing $Q$ then the subspace $\mathbf{M} V^{i}=O$ of $P G(5, \mathscr{F})$ is a plane.

Thus each point in $\mathbf{S}$ may be thought of as simultaneously representing both a plane in $\mathscr{H}$ and a plane in $\mathscr{K}$.

2.6. The collineations $A, B, C$ and $R$. Now consider the projective collineations $A, B, C$ and $R$ of $P G(3, \mathscr{F})$ with matrices $\mathbf{A}, \mathbf{B}, \mathbf{C}$ and $\mathbf{R}$ (respectively). The calculations reported in Section 2.4 show that the action of the collineations $a, b, c$ and $r$ on the set $\mathscr{H}$ may be determined by examining the actions of $A, B, C^{-1}$ and $R$ (respectively) on the set $\mathbf{S}$; and that the actions of $a, b, c$ and $r$ on the set $\mathscr{K}$ may be determined by examining the actions of $A, B, C$ and $R$ (respectively) on $\mathbf{S}$.

2.7. The twenty points in $\mathbf{S}$. Observing the zeros on the diagonal of $\mathbf{M}$, we immediately identify four points belonging to $\mathrm{S}$, namely the points $P_{11}, P_{12}, P_{13}, P_{14}$ represented by the vectors $(1,0,0,0),(0,1,0,0),(0,0,1,0),(0,0,0,1)$. The corresponding planes in $\mathscr{H}$ are obtained by setting the rows (one at a time) of $\mathbf{M}$ equal to zero, whereas the corresponding planes in $\mathscr{K}$ are obtained by setting the columns of $\mathbf{M}$ equal to zero.

Now let $F=R^{-1} C R A$ and $P_{m n}=P_{1 n} F^{m-1}$ (for $m=1, \ldots, 5$ and $\left.n=1, \ldots, 4\right)$. 
It can be checked that the points $P_{m n}$ are given by the vectors:

$$
\begin{aligned}
& (1,0,0,0),(0,1,0,0),(0,0,1,0),(0,0,0,1), \\
& (-1,1,-1,1),(-1,-1,1,1),(1,1,1,1),(1,-1,-1,1), \\
& (-i,-i,-1,1),(i, i,-1,1),(-i, i, 1,1),(i,-i, 1,1), \\
& (-i,-1,-i, 1),(i, 1,-i, 1),(-i, 1, i, 1),(i,-1, i, 1), \\
& (1, i,-i, 1),(1,-i, i, 1),(-1, i, i, 1),(-1,-i,-i, 1) .
\end{aligned}
$$

Here the vector in row $m$, column $n$ represents the point $P_{m n}$.

We have found 20 points in $S$. It can be checked that there are no further points in $\mathrm{S}$ as follows. Suppose $U=(X, Y, Z, T)$ is a vector representing a point $P$ in $\mathbf{S}$. Then the four entries of $U \mathbf{M}$ are linearly dependent over $\mathscr{F}$. It follows (cf. [5, pp. 79-80]) that all the $4 \times 4$ minors of the matrix

$$
\left(\begin{array}{cccccc}
0 & T & Z & 0 & 0 & -Y \\
0 & -Z & 0 & T & X & 0 \\
Y & 0 & 0 & -X & 0 & T \\
-X & 0 & -Y & 0 & -Z & 0
\end{array}\right)
$$

vanish. Solving the corresponding system of equations, we find that $P$ is one of the points $P_{m n}$. We could of course have found the points $P_{m n}$ in the first place by simply solving the system of equations just referred to.

2.8. The group $\mathscr{G}$. It can easily be checked that the group $\langle A, B, C, R\rangle$ acts transitively on $\mathbf{S}$, from which it follows (in view of the remarks in Section 2.6) that the group $\langle a, b, c, r\rangle$ acts transitively on each of $\mathscr{H}$ and $\mathscr{K}$. Moreover, j swaps $\mathscr{H}$ and $\mathscr{K}$. (See section 2.4.) So the group $\mathscr{G}=\langle a, b, c, r, j\rangle$ acts transitively on the set $\mathscr{H} \cup \mathscr{K}$, and each element of $\mathscr{G}$ either maps $\mathscr{H}$ onto itself and $\mathscr{K}$ onto itself or else swaps $\mathscr{H}$ and $\mathscr{K}$.

2.9. Let us denote by $H_{m n}$ the plane in $\mathscr{H}$, and by $K_{m n}$ the plane in $\mathscr{K}$, corresponding to the point $P_{m n}$ (for $m=1, \ldots, 5$ and $n=1, \ldots, 4$ ).

Now it is clear that $H_{14}$ does not intersect $K_{14}$. It follows, by the argument expounded in [5, pp. 72-73], that $H_{14}$ intersects every other plane $K_{m n}$. In fact it is not difficult to check (using explicit equations for the planes $H_{m n}$ and $K_{m n}$ ) that $H_{14}$ intersects each of $K_{11}, K_{12}$ and $K_{13}$ in a line; that it intersects each of the planes $K_{m n}$ with $m \neq 1$ in a point; and that it intersects each of the planes $H_{11}, H_{12}$ and $H_{13}$ in a point, but does not intersect any of the planes $H_{m n}$ with $m \neq 1$.

2.10. The facts listed in Sections 2.8 and 2.9 suffice to show that $(\mathscr{H}, \mathscr{K})$ is a transitive non-degenerate double-twenty of planes. The pairing map $\pi: \mathscr{H} \longmapsto \mathscr{K}$ is given by the rule $\pi(H)=$ the unique plane in $\mathscr{K}$ which does not intersect $H$. 


\section{The geometry of the set $S$}

3.1. Let $\mathbf{T}_{m}=\left\{P_{m 1}, P_{m 2}, P_{m 3}, P_{m 4}\right\}$ (for $m=1, \ldots, 5$ ). Then simple calculations show that each of the collineations $A, B, C$ and $R$ permutes the five sets $\mathbf{T}_{1}, \ldots, \mathbf{T}_{5}$, inducing on $\left\{\mathbf{T}_{1}, \ldots, \mathbf{T}_{5}\right\}$ the permutations $\left(\mathbf{T}_{1}, \mathbf{T}_{2}\right)\left(\mathbf{T}_{3}, \mathbf{T}_{5}\right)\left(\mathbf{T}_{4}\right),\left(\mathbf{T}_{1}\right)\left(\mathbf{T}_{2}\right)\left(\mathbf{T}_{3}\right)\left(\mathbf{T}_{4}\right)\left(\mathbf{T}_{5}\right)$, $\left(\mathbf{T}_{1}\right)\left(\mathbf{T}_{2}, \mathbf{T}_{4}\right)\left(\mathbf{T}_{3}, \mathbf{T}_{5}\right)$ and $\left(\mathbf{T}_{1}\right)\left(\mathbf{T}_{2}\right)\left(\mathbf{T}_{3}, \mathbf{T}_{4}, \mathbf{T}_{5}\right)$ respectively. The permutation group induced by the collineation group $\langle A, B, C, R\rangle$ on the set $\left\{\mathbf{T}_{1}, \ldots, \mathbf{T}_{5}\right\}$ is the alternating group on that set. The collineation $F=R^{-1} C R A$ used to label the points $P_{m n}$ induces the 5-cycle $\left(\mathbf{T}_{1}, \mathbf{T}_{2}, \mathbf{T}_{3}, \mathbf{T}_{4}, \mathbf{T}_{5}\right)$ on $\left\{\mathbf{T}_{1}, \ldots, \mathbf{T}_{5}\right\}$.

Each of $B, C$ and $R$ maps $\mathbf{T}_{1}$ onto itself. It is easily checked that the permutation group induced by $\langle B, C, R\rangle$ on $\mathbf{T}_{1}$ is the alternating group on $\mathbf{T}_{1}$.

3.2. Now the points $P_{11}, P_{12}, P_{13}, P_{14}$ are clearly non-coplanar, since they are represented by the vectors $(1,0,0,0),(0,1,0,0),(0,0,1,0),(0,0,0,1)$. So $\mathbf{T}_{1}$ is the vertex-set of a tetrahedron in $P G(3, \mathscr{F})$. It follows that each of $\mathbf{T}_{2}, \mathbf{T}_{3}, \mathbf{T}_{4}$ and $\mathbf{T}_{5}$ is also the vertex set of a tetrahedron.

It turns out that the five tetrahedra $\mathbf{T}_{1}, \ldots, \mathbf{T}_{5}$ are very specially related in that any two of them form a desmic pair (that is, are such that each edge of either intersects two opposite edges of the other). This is easily verified for the pair $\mathbf{T}_{1}, \mathbf{T}_{2}$. Since $\langle A, B, C, R\rangle$ acts doubly transitively on $\left\{\mathbf{T}_{1}, \ldots, \mathbf{T}_{5}\right\}$, it then follows for the other pairs.

The five tetrahedra $\mathbf{T}_{1}, \ldots, \mathbf{T}_{5}$ may be used to construct ten further tetrahedra, since (cf. Hudson [2, pp. 1-3]) any desmic pair is part of a triple of mutually desmic tetrahedra. It can be shown that the vertices, edges and faces of the resulting system of 15 tetrahedra are the points, lines and planes of a Klein $60_{15}$ configuration consisting of 60 points, 30 lines and 60 planes with 15 points in each plane, 15 planes through each point, 6 points on each line and 6 planes through each line (cf. [2, pp. 42-43 and pp. 49-50]).

\section{The group $\mathscr{G}$ and the set of planes $\mathscr{H} \cup \mathscr{K}$}

4.1. Let $\mathscr{H}_{m}=\left\{H_{m 1}, H_{m 2}, H_{m 3}, H_{m 4}\right\}, \mathscr{K}_{m}=\left\{K_{m 1}, K_{m 2}, K_{m 3}, K_{m 4}\right\}$ and $\mathscr{O}_{m}=$ $\mathscr{K}_{m} \cup \mathscr{K}_{m}$ (for $m=1, \ldots, 5$ ). Then from the remarks in Sections 2.6 and 3.1, and the fact that $C$ and $C^{-1}$ induce the same permutation on $\left\{\mathbf{T}_{1}, \ldots, \mathbf{T}_{5}\right\}$, we deduce that the collineation group $\langle a, b, c, r\rangle$ acts as a permutation group on each of the sets $\left\{\mathscr{H}_{1}, \ldots, \mathscr{H}_{5}\right\},\left\{\mathscr{K}_{1}, \ldots, \mathscr{K}_{5}\right\}$ and $\left\{\mathscr{O}_{1}, \ldots, \mathscr{O}_{5}\right\}$, inducing in each case the alternating group on the set.

Now $j$ swaps $H_{m n}$ and $K_{m n}$ for all $m$ and $n$ (cf. Section 2.4). It follows that $\left(\mathscr{O}_{m}\right) j=\mathscr{O}_{m}$ for all $m$. So $\mathscr{G}=\langle a, b, c, r, j\rangle$ acts on the set $\left\{\mathscr{O}_{1}, \ldots, \mathscr{O}_{5}\right\}$, and the induced permutation group is the alternating group on $\left\{\mathscr{O}_{1}, \ldots, \mathscr{O}_{5}\right\}$. 
4.2. The equations of $H_{11}, \ldots, H_{14}, K_{11}, \ldots, K_{14}$ can be obtained immediately by looking at the rows and columns of $\mathbf{M}$. From these it is easy to check that the configuration, consisting of the 8 planes of $\mathscr{O}_{1}$ together with the 12 lines and 6 points obtained from them by forming intersections, is isomorphic (as an incidence structure) to the configuration consisting of the faces, edges and vertices of a regular octahedron in 3-dimensional Euclidean space. It is also easy to check that each of the three 'diagonal lines' of $\mathscr{O}_{1}$ passes through two of the six points $Y_{1}, \ldots, Y_{6}$ represented by the vectors $(1,1,0,0,0,0),(-1,1,0,0,0,0),(0,0,1,1,0,0),(0,0,-1,1,0,0)$, $(0,0,0,0,1,1),(0,0,0,0,-1,1) ;$ and that each of the collineations $a, b, c, r$ and $j$ permutes $Y_{1}, \ldots, Y_{6}$.

It follows, since $\mathscr{G}$ acts transitively on $\left\{\mathscr{O}_{1}, \ldots, \mathscr{O}_{5}\right\}$ and each element of $\mathscr{G}$ maps $\left\{Y_{1}, \ldots, Y_{6}\right\}$ onto itself, that each of the sets $\mathscr{O}_{m}$ gives rise to a configuration isomorphic to a regular octahedron in 3-dimensional Euclidean space, and that each of the three 'diagonal lines' of $\mathscr{O}_{m}$ passes through two of the six points $Y_{1}, \ldots, Y_{6}$.

Now consider the pairing map $\pi: \mathscr{H} \longmapsto \mathscr{K}$ of the double-twenty $(\mathscr{H}, \mathscr{K})$. Recall that $\left(H_{14}\right) \pi=K_{14}$ (cf. Sections 2.9 and 2.10), that each of $a, b, c$ and $r$ maps each of the sets $\mathscr{H}, \mathscr{K}$ and $\left\{\mathscr{O}_{1}, \ldots, \mathscr{O}_{5}\right\}$ onto itself, and that $\langle a, b, c, r\rangle$ acts transitively on $\mathscr{H}$. It follows that if $H$ belongs to $\mathscr{H}_{m}$ then

$$
H \pi=\text { the unique plane in } \mathscr{O}_{m} \text { which does not intersect } H \text {. }
$$

In other words, $H$ and $H \pi$ correspond to a pair of opposite faces of the octahedron referred to in the previous paragraph.

4.3. The representation of $\mathscr{G}$ as a permutation group on $\left\{\mathscr{O}_{1}, \ldots, \mathscr{O}_{5}\right\}$, described in Section 4.1, is not faithful. Indeed it is a straight-forward exercise (in view of the very simple form of the equations for the planes in $\mathscr{O}_{1}$ ) to verify that the kernel of this representation is the (normal) subgroup $\mathscr{N}=\left\langle b, r b r^{-1}, c^{2}, r c^{2} r^{-1}, j\right\rangle$ of $\mathscr{G}$. Since $\mathscr{G} / \mathscr{N} \cong A_{5}$ and $\mathscr{N}$ is an elementary abelian 2-group of order 32 , it follows that $|\mathscr{G}|=32 \times 60=1920$.

We are interested primarily, of course, in the action of $\mathscr{G}$ on $\mathscr{H} \cup \mathscr{K}$. This representation is faithful (and transitive). Examining the action of $\langle A, B, C, R\rangle$ on S, we see that the orbits (in $\mathscr{H} \cup \mathscr{K}$ ) of the stabilizer of $H_{14}$ in $\mathscr{G}$ are $\left\{H_{14}\right\},\left\{K_{14}\right\}$, $\left\{H_{11}, H_{12}, H_{13}\right\},\left\{K_{11}, K_{12}, K_{13}\right\},\left\{H_{m n}: m \neq 1\right\}$ and $\left\{K_{m n}: m \neq 1\right\}$.

\section{The self-polar case}

5.1. Let us now suppose that our coordinate field $\mathscr{F}$ satisfies (in addition to the two conditions imposed in Section 2.1) the condition that it possesses an involutory automorphism $\theta$ which swaps $i$ and $-i$. Then we may consider the unitary polarity 
$\alpha$ in $P G(5, \mathscr{F})$ which, for all non-zero vectors $\mathbf{X}_{\circ}=\left(x_{\circ}, u_{\circ}, y_{\circ}, v_{\circ}, z_{\circ}, w_{\circ}\right)$ in $\mathscr{F}^{6}$, swaps the point represented by the vector $\mathbf{X}_{\circ}$ with the hyperplane whose equation is

$$
x_{\circ}{ }^{\theta} x+u_{\circ}{ }^{\theta} u+y_{\circ}{ }^{\theta} y+v_{\circ}{ }^{\theta} v+z_{\circ}{ }^{\theta} z+w_{\circ}{ }^{\theta} w=0 .
$$

A few simple calculations show that $\alpha$ commutes with each of the collineations $a, b, c, r$ and $j$, from which it follows that $\alpha$ commutes with every element of $\mathscr{G}$.

Now each element of $\langle a, b, c, r\rangle$ maps $\mathscr{H}$ onto $\mathscr{H}$ and $\mathscr{K}$ onto $\mathscr{K}$, and therefore commutes with the pairing map $\pi: \mathscr{H} \longmapsto \mathscr{K}$. Also $\langle a, b, c, r\rangle$ acts transitively on $\mathscr{H}$, each element of $\langle a, b, c, r\rangle$ commutes with $\alpha$ and $\left(H_{14}\right) \alpha=K_{14}=\left(H_{14}\right) \pi$. It follows that $H \alpha=H \pi$ for all $H$ in $\mathscr{H}$. In other words, the (transitive non-degenerate) double-twenty $(\mathscr{H}, \mathscr{K})$ is self-polar with respect to the unitary polarity $\alpha$.

\section{References}

[1] P. Dembowski, Finite geometries, Ergebnisse Math. 44 (Springer, Berlin, 1968).

[2] R. W. H. T. Hudson, Kummer's quartic surface (Cambridge University Press, Cambridge, 1905). Reprinted, 1990.

[3] P. Kirkpatrick, 'Self-polar double-N's defined by certain pairs of normal rational curves', J. Aust. Math. Soc. 6 (1966), 210-220.

[4] _ , 'A construction for a self-polar double- $\mathrm{N}$ associated with a pair of normal rational curves', J. Aust. Math. Soc. 8 (1968), 415-422.

[5] T. G. Room, The geometry of determinantal loci (Cambridge University Press, Cambridge, 1938).

[6] —_ 'Self-polar double configurations, I and II', J. Aust. Math. Soc. 5 (1965), 65-68, 69-75.

[7] W. R. Scott, Group theory (Prentice-Hall, Englewood Cliffs, 1964).

School of Mathematics and Statistics

University of Sydney, NSW 2006

Australia 